DAMTP R-98/02

hep-th/9801127

\title{
Bound States of D-Branes and the Non-Abelian Born-Infeld Action
}

\author{
D. Brecher" and M. J. Perry \\ D.A.M.T.P., University of Cambridge, Cambridge CB3 9EW, U.K.
}

\begin{abstract}
We attempt to settle the issue as to what is the correct non-abelian generalisation of the Born-Infeld action, via a consideration of the two-loop $\beta$-function for the non-abelian background gauge field in open string theory. An analysis of the bosonic theory alone shows the recent proposal of Tseytlin's [1] to be somewhat lacking. For the superstring, however, this proposal would seem to be correct, and not just within the approximation used in [1]. Since it is this latter case that is relevant to the description of D-branes we, in effect, obtain an independent verification of Tseytlin's result. Some issues involved in the concept of non-abelian T-duality are discussed; and it is shown how the interaction between separated and parallel branes, in the form of massive string states, emerges.
\end{abstract}

\footnotetext{
${ }^{1}$ email d.r.brecher@damtp.cam.ac.uk.

${ }^{2}$ email malcolm@damtp.cam.ac.uk.
} 


\section{Introduction}

The connections between D-branes [2, 3, 4] and the non-linear electrodynamics of Born and Infeld [5] are well-known. The pure Born-Infeld (BI) action was found to describe the low energy effective field theory of open bosonic string theory in [6], using the background field technique applied to the two-dimensional $\sigma$-model. These methods were extended to an analysis of a Dirichlet $\sigma$-model in [7], where the Dirac-Born-Infeld (DBI) action was found to describe the world-volume theory of the $\mathrm{D} p$-brane; this latter can be deduced easily by a consideration of T-duality as applied to the BI theory, as we will review below. Such a theory is one of a non-linear electrodynamics on the brane, the transverse coordinates of which are T-dual to the world-volume (abelian) gauge field. It is natural to ask what is the non-abelian generalisation of this theory, and there has been some recent [1, 8], and some not so recent [9, 10], work addressing this question. The issue has not been resolved fully, however; and it is at just such a resolution that this paper is aimed.

By attaching charges, the Chan-Paton factors, to the ends of open strings, we generate a spacetime gauge theory. By restricting the strings to end on a $(p+1)$-dimensional hypersurface, the Dp-brane, we have a gauge theory on the world-volume of the brane. Since open strings come in two different types, oriented and unoriented, there are different possible gauge theories: a $U(N)$ theory for the oriented string; and an $S O(N)$ or $U S p(N)$ theory for the unoriented string. Within D-brane theory, the former is of interest because a $U(N)$ gauge theory is thought to describe a collection of $N \mathrm{D} p$-branes [11]. This is observed easily: if we have $N$ separated and parallel $\mathrm{D} p$-branes, there will be massless open strings ending on each brane, giving a $U(1)^{N}$ gauge theory. There will also be open strings stretched between the branes, however, with a mass proportional to their length. As the branes are brought together, these strings become massless, extending the resulting gauge group to $U(N)$. Since, roughly speaking, $U(N) \sim U(1) \times S U(N)$, such a theory will be one of a bound state of such D-branes, the $U(1)$ factor corresponding to the collective coordinate; and the action describing such states ought, then, to be a non-abelian generalisation of the DBI action. Interestingly, if we regard this bound state as a 'brane' in its own right, we see that its world--volume theory will contain a non-abelian gauge field; and the transverse coordinates of the 'brane' will be T-dual to this. That is, they will themselves become matrix-valued, a notion which has lead to the Matrix description of M-Theory [12]. By deciding upon what we believe to be the correct non-abelian BI action, we hope to be able to answer some of 
the questions which arise in this context.

Since we will employ the background field technique we give, in section two, a very brief description of how this method has been used in open bosonic string theory to derive the results pertaining to D-branes. In section three we consider a Yang-Mills background field, and calculate the $\beta$-function for this field to two-loops, following closely the analysis of [10]. Since D-branes are embedded in superstring theory, as opposed to the purely bosonic theory, we then extend these results to a consideration of the open superstring (and thereby provide an explanation for why the aforementioned purely bosonic results carry through to the full-blown supersymmetric theory). In section four we consider the possible non-abelian generalisations of the BI action, finding one which gives an equation of motion consistent with the results of section three only. Finally, we make some comments as to the nature of non-abelian T-duality; the first steps towards explaining the appearance of matrix-valued spacetime coordinates. For separated and parallel branes, we should see an interaction between them in the form of massive strings. To lowest order this will emerge as a massive Yang-Mills theory; and we show how this comes about. We offer further a few remarks concerning D4-branes with an (anti-)self-dual field strength; one of the few obvious cases for which the non-abelian BI action is tractable.

\section{Background Field Method in Open String Theory}

It is well-known that consistency of tree-level string theory determines the equations of motion of the background spacetime in which the string lives, and of the matter fields therein; for an extensive review, see [14]. The $\sigma$-model is a theory of two-dimensional massless scalar fields, the spacetime coordinates, living on the string worldsheet; the 'coupling constants' of the model being the metric $G_{a b}$, the two-form $B_{a b}$, the dilaton $\Phi$ and, for open strings, the gauge field $A_{a}$. Two-dimensional loop effects contain logarithmic divergences, so these couplings must be renormalised. The requirement that this renormalisation be scaleindependent is just the requirement that the $\beta$-function for each field vanish. From a stringy perspective, however, the couplings are just the classical spacetime fields in which the string

\footnotetext{
${ }^{3}$ There are two ways of looking at this: on the one hand, conformal invariance of the string theory is the requirement that the trace of the energy-momentum tensor vanish i.e., that the Weyl anomaly coefficients are zero. This is how the results of, e.g. [13], were found. On the other, scale invariance is the requirement that the renormalisation group $\beta$-functions vanish. We are dealing here with the latter approach although this will not effect the generality of our results, since there is a well-defined procedure for generating the anomaly coefficients from the $\beta$-functions, and vice versa [14].
} 
lives; condensates of the massless string states. The statement $\beta^{(A)}=0$ is, then, interpreted as an equation of motion for the spacetime field $A_{a}(X)$.

The background field method consists of splitting the embeddings $X^{a}$ into a classical and a quantum piece,

$$
X^{a}(\tau)=\bar{X}^{a}(\tau)+\xi^{a}(\tau)
$$

thereby constructing a two-dimensional quantum field theory in the variable $\xi^{a}(\tau) . \tau$ is the time coordinate on the worldsheet, only the $\tau$ dependence being relevant here, since all gauge field interactions occur on the boundary of the worldsheet. We work in flat spacetime, thereby avoiding the geometrical considerations concerning the identification of the quantum field as a normal coordinate [15]; and, since we are interested in divergences alone, we work with a euclidean target space and worldsheet. Expanding the usual string action $S[X]=S[\bar{X}+\xi]$ about an arbitrary on-shell reference configuration $\bar{X}^{a}$, the functional generating all loop diagrams with the external legs amputated is

$$
\Omega[X]=\int D \xi \exp (-(S[\bar{X}+\xi]-S[\bar{X}]))
$$

with $\hbar=1$. Following [15] we work using time-dependent perturbation theory, writing

$$
\Omega[X]=\langle 0| \exp (-(S[\bar{X}+\xi]-S[\bar{X}))|0\rangle
$$

Then the effective action is

$$
\Gamma[X]=-\ln \Omega[X]=\langle 0|(S[\bar{X}+\xi]-S[\bar{X}])| 0\rangle
$$

All possible loop diagrams are contained in (2), some of which will be divergent. To cancel these divergences, counterterms must be added to the action: $S[X] \rightarrow S[X]+\triangle S$; and it is via the addition of such counterterms that the spacetime fields get renormalised.

These techniques were used in [6] to deal with the open bosonic string in a background Maxwell field. It was found that the solutions of the equation of motion following from the BI lagrangian,

$$
\mathcal{L}_{B I}=\sqrt{\operatorname{det}\left(\delta_{a b}+2 \pi \alpha^{\prime} F_{a b}\right)}
$$

are just the solutions of the condition for scale invariance, $\beta_{a}^{A}=\alpha^{\prime}\left(1-\left(2 \pi \alpha^{\prime} F\right)^{2}\right)^{-1 b c} \partial_{(b} F_{c) a}=$ 0 :

$$
\left.\frac{\delta \mathcal{L}_{B I}}{\delta A_{a}}=\sqrt{\operatorname{det}\left(\delta_{a b}+2 \pi \alpha^{\prime} F_{a b}\right.}\right)\left(1-\left(2 \pi \alpha^{\prime} F\right)^{2}\right)_{a}^{-1 b} \beta_{b}^{A}=0
$$


Indeed, as we will comment on below, this is true for the superstring also [16]. This result is valid to all orders in $\alpha^{\prime}$, the two-dimensional loop counting parameter, although is exact for a slowly-varying field strength only; in the generic case, there will presumably be corrections depending on derivatives of the field strength. In the non-abelian case, it does not appear to be possible to obtain an analogously exact (in $\alpha^{\prime}$ ) result, and so we work to the twoloop order only. Moreover, and as we will discuss further below, it is unclear as to how the variation, analogous to (幽), carries through to the non-abelian case. At the order to which we are working, however, the analysis would seem to be valid.

We note here that we will in fact take the BI lagrangian to be slightly different to (3): for a euclidean target space we have

$$
\mathcal{L}_{B I}=\left(2 \pi \alpha^{\prime} g\right)^{-2}\left[1-\sqrt{\operatorname{det}\left(\delta_{a b}+2 \pi \alpha^{\prime} g F_{a b}\right)}\right],
$$

where $2 \pi \alpha^{\prime}$ is the inverse string tension, and we reintroduced the gauge coupling $g$. This differs from (3) only in that it is zero for a vanishing field strength.

The pure BI Lagrangian (5) describes a low energy effective spacetime theory in which the effects of gravity are ignored: the open strings, having electromagnetic charges at their ends, generate a ten-dimensional spacetime gauge theory. To make contact with the concept of the D-brane, we must consider T-dualising this theory [4]. That is, if we compactify the theory on a circle of radius $R$ in, say, the $X^{9}$ direction, we can reformulate it in terms of the dual coordinate, $\tilde{X}^{9}$. Now, however, we find the ends of the strings to lie on the same plane, $\tilde{X}^{9}(\pi)-\tilde{X}^{9}(0)=2 \pi n \alpha^{\prime} / R=2 \pi n \tilde{R}$ : a D-brane has appeared on the dual circle, of radius $\tilde{R}=\alpha^{\prime} / R$. Moreover, we have that $\tilde{X}^{9}=2 \pi \alpha^{\prime} g A_{9}$ : the gauge field in the compact direction is $\mathrm{T}$-dual to the transverse coordinate of the $\mathrm{D}$-brane. In the dual picture, then, we have $F_{a 9}=\left(2 \pi \alpha^{\prime} g\right)^{-1} \partial_{a} \tilde{X}_{9}, \quad a, b \neq 9$. Using the identity [17

$$
\operatorname{det}\left(\begin{array}{cc}
N & -A^{t} \\
A & M
\end{array}\right)=\operatorname{det}(M) \operatorname{det}\left(N+A^{t} M^{-1} A\right)=\operatorname{det}(N) \operatorname{det}\left(M+A N^{-1} A^{t}\right)
$$

with $t$ denoting transposition, we have the well-known result

$$
\mathcal{L}_{D B I}=\left(2 \pi \alpha^{\prime} g\right)^{-2}\left[1-\sqrt{\operatorname{det}\left(\delta_{a b}+\partial_{a} X^{9} \partial_{b} X^{9}+2 \pi \alpha^{\prime} g F_{a b}\right)}\right],
$$

where we have dropped the tilde on the dual coordinate. This is the DBI Lagrangian in the so-called static gauge, describing a D8-brane, $X^{9}$ being its transverse coordinate; $\delta_{a b}+$ $\partial_{a} X^{9} \partial_{b} X^{9}$ is just the pull-back to the worldvolume of the spacetime metric. It is important 
to realise that this is the $\mathrm{T}$-dual of the pure BI spacetime theory (5). In the same way, the ten-dimensional non-abelian generalisation of the BI theory will describe an effective spacetime non-abelian gauge theory; and it is only through $\mathrm{T}$-dualising this that we will find bound states of D-branes.

\section{$3 \beta$-Functions for Pure Yang-Mills Backgrounds}

Since we are not concerned with effects in the bulk of the worldsheet, we set $\Phi=B_{a b}=0$. The action for open superstrings coupled to a Yang-Mills spacetime field $A_{a}(X)$ is then

$$
\begin{aligned}
S & =S_{\Sigma}+S_{\partial \Sigma}, \\
S_{\Sigma} & =\frac{1}{4 \pi \alpha^{\prime}} \int_{\Sigma} d^{2} \sigma\left[\sqrt{\gamma} \gamma^{\mu \nu} \partial_{\mu} X^{a} \partial_{\nu} X_{a}-\frac{i}{2} \bar{\Psi}^{a} \rho^{\mu} \partial_{\mu} \Psi_{a}\right], \\
S_{\partial \Sigma} & =-\ln \operatorname{Tr} \mathcal{P} \exp \left(i g \oint_{\partial \Sigma} d \tau\left[A_{a}(X) \partial_{\tau} X^{a}-\frac{1}{2} \psi^{a} \psi^{b} F_{a b}(X)\right]\right) \\
& =-\ln \operatorname{Tr} \mathcal{P}(U[A]),
\end{aligned}
$$

where $-\infty<\tau<\infty, 0 \leq \sigma \leq \pi, \mu$ is a worldsheet index, $\rho^{\mu}$ being the two-dimensional Dirac matrices, and $a, b=0, \ldots, 9$. The spinor $\psi^{a}(\sigma, \tau)$ in (9) is just the restriction to the boundary of the usual worldsheet Majorana spinor $\Psi^{a}(\sigma, \tau): \psi^{a}=\left.\Psi^{a}\right|_{\partial \Sigma}$. That is, it depends only on the combination of the left and right moving fermions which is not set to zero by the boundary conditions. The gauge field $A_{a}(X)=A_{a}^{i}(X) t^{i}$ takes values in the fundamental representation of the group algebra, which we leave unspecified for the time being, and $F_{a b}(X)=\partial_{a} A_{b}-\partial_{b} A_{a}-i g\left[A_{a}, A_{b}\right]$ is the non-abelian field strength. The factor of $i$ in (9) is necessary if $\left\{t^{i}\right\}$ is to be an hermitian basis of the group algebra. $\mathcal{P}$ is the path-ordering operation and $\operatorname{Tr} \mathcal{P}(U[A])$ is the supersymmetrised Wilson loop. The pathordered exponential reduces to a standard exponential if the matrices at different spacetime positions commute i.e., if the gauge group is abelian; in which case we would have the usual $S_{\partial \Sigma}=-i g \oint_{\partial \Sigma} d \tau\left[A_{a}(X) \partial_{\tau} X^{a}-\frac{1}{2} \psi^{a} \psi^{b} F_{a b}(X)\right]$.

We must note that the background-quantum split (1) is to be applied to the (bosonic) embeddings only, the fermionic variable $\psi^{a}$ being treated as a quantum field from the outset. Making this split, then, and expanding (9) with respect to $\xi^{a}$ we have

$$
\begin{gathered}
\exp \left(-\left(S_{\partial \Sigma}[\bar{X}+\xi]-S_{\partial \Sigma}[\bar{X}]\right)\right)=\operatorname{Tr} \mathcal{P}(U[A(\bar{X}+\xi)]-U[A(\bar{X})])= \\
i g \oint_{\partial \Sigma} d \tau \operatorname{Tr} \mathcal{P}\left(U [ A ] \left[F_{a b} \xi^{a} \partial_{\tau} \bar{X}^{b}-\frac{1}{2} F_{a b} \psi^{a} \psi^{b}-\frac{1}{2} D_{a} F_{b c} \xi^{a} \psi^{b} \psi^{c}+\frac{1}{2} D_{b} F_{a c} \xi^{a} \xi^{b} \partial_{\tau} \bar{X}^{c}\right.\right.
\end{gathered}
$$




$$
\begin{gathered}
+\frac{1}{2} F_{a b} \xi^{a} \partial_{\tau} \xi^{b}-\frac{1}{4} D_{a} D_{b} F_{c d} \xi^{a} \xi^{b} \psi^{c} \psi^{d}+\sum_{n=3}^{\infty}\left(\frac{1}{n !} D_{a_{1}} \ldots D_{a_{n-1}} F_{a_{n} b} \xi^{a_{1}} \ldots \xi^{a_{n}} \partial_{\tau} \bar{X}^{b}\right. \\
\left.\left.\left.+\frac{n-1}{n !} D_{a_{1}} \ldots D_{a_{n-2}} F_{a_{n-1} a_{n}} \xi^{a_{1}} \ldots \xi^{a_{n-1}} \partial_{\tau} \xi^{a_{n}}-\frac{1}{2} \frac{1}{n !} D_{a_{1}} \ldots D_{a_{n}} F_{b c} \xi^{a_{1}} \ldots \xi^{a_{n}} \psi^{b} \psi^{c}\right)\right]\right)
\end{gathered}
$$

where $D_{a}$ is the gauge covariant derivative, and all spacetime fields are evaluated at $\bar{X}^{a}$. We have kept the $F_{a b} \psi^{a} \psi^{b}$ term for the reasons mentioned above. We include the derivation of (10) in an Appendix for completeness (see, e.g., 18, 19]). We will ignore the $\mathcal{O}(\xi)$ terms which vanish on-shell, thereby simplifying our analysis as compared to that of [10]. All interactions relevant to $\beta^{(A)}$ are contained in the expansion (10), via the expression (2) for the effective action; those interactions occurring in the interior of the worldsheet, generated by (8), effect a renormalisation of the spacetime metric only.

\subsection{The Bosonic Sector}

Setting the fermions to zero, we have $\exp \left(-S_{\partial \Sigma}[X]\right)=\operatorname{Tr} \mathcal{P}(U[A])$ being the standard Wilson loop. We cannot define the exact propagator of the theory, due to the path-ordering involved in this boundary term which is why, in contrast to the abelian case, we cannot work to all orders in $\alpha^{\prime}$, even for a slowly-varying field strength. Expanding the interior action (8) with respect to $\xi^{a}$ and requiring the $\mathcal{O}(\xi)$ term to vanish gives the usual equation of motion $\partial_{\mu}\left(\sqrt{\gamma} \gamma^{\mu \nu} \partial_{\nu}\right) \bar{X}^{a}=0$ and Neumann boundary condition $\left.\partial_{n} \bar{X}^{a}\right|_{\partial \Sigma}=0$. Due to conformal invariance of the classical theory, we are free to work on the unit disc, with the coordinates $z=\tau+i \sigma$ and $\bar{z}=\tau-i \sigma$. Then the propagator $G^{a b}\left(z, z^{\prime}\right)=\left\langle 0\left|T\left[\xi^{a}(z) \xi^{b}\left(z^{\prime}\right)\right]\right| 0\right\rangle=$ $-2 \pi \alpha^{\prime} \delta^{a b} N\left(z, z^{\prime}\right)$ where $N\left(z, z^{\prime}\right)$ is the Neumann function on the disc,

$$
N\left(z, z^{\prime}\right)=\frac{1}{2 \pi} \ln \left(\left|z-z^{\prime}\right|\left|z-\bar{z}^{\prime-1}\right|\right)
$$

satisfying $\partial_{\mu}\left(\sqrt{\gamma} \gamma^{\mu \nu} \partial_{\nu}\right) N\left(z, z^{\prime}\right)=\delta^{2}\left(z-z^{\prime}\right)$.

On the boundary of the worldsheet we can work with the single angular variable $\theta, 0 \leq$ $\theta \leq 2 \pi$, and we have 20]

$$
\begin{aligned}
N\left(z, z^{\prime}\right)=N\left(e^{i \theta}, e^{i \theta^{\prime}}\right) & =\frac{1}{\pi} \ln \left|z-z^{\prime}\right| \\
& =\frac{1}{2 \pi} \ln (2-2 \cos \beta), \\
& =-\frac{1}{\pi} \sum_{n=1}^{\infty} \frac{\cos n \beta}{n} e^{-\varepsilon n},
\end{aligned}
$$


where $\beta=\theta-\theta^{\prime}$ and $\varepsilon$ is an ultra-violet cut-off [1]. Note that we need not concern ourselves over infra-red divergences: since they can be regulated via $G \sim \ln \left(\mu\left|z-z^{\prime}\right|\right), \mu$ an infra-red cut-off, they will not contribute in the limit $z \rightarrow z^{\prime}$.

Counterterms must be added to (9), then, to cancel the ultra-violet divergences arising from the propagator $G^{a b}(0)=-2 \pi \alpha^{\prime} \delta^{a b} N(0)=-2 \pi \alpha^{\prime} \delta^{a b}\left(N_{d i v}+N_{f i n}\right)$ with, from (12), $N_{d i v}=\left.\frac{1}{\pi} \ln (\varepsilon)\right|_{\varepsilon \rightarrow 0}$. From (9), these counterterms must take the form

$$
\exp (-\triangle S)=\operatorname{Tr} \mathcal{P}(U[A+\delta A]-U[A])
$$

Since $G^{a b} \sim \alpha^{\prime}, \alpha^{\prime}$ is the loop counting parameter for the two-dimensional field theory, and we write

$$
\delta A_{a}=\alpha^{\prime} \delta_{1} A_{a}+\alpha^{\prime 2} \delta_{2} A_{a}+\mathcal{O}\left(\alpha^{\prime 3}\right)
$$

Then the counterterms are generated via

$$
\begin{gathered}
\exp (-\triangle S)=\operatorname{Tr} \mathcal{P}\left(U\left[A+\alpha^{\prime} \delta_{1} A+\alpha^{\prime 2} \delta_{2} A\right]-U[A]\right)= \\
i \alpha^{\prime} \int_{0}^{2 \pi} d \theta \operatorname{Tr} \mathcal{P}\left(U[A] \delta_{1} A_{a} \partial_{\theta} \bar{X}^{a}\right)+i \alpha^{\prime 2} \int_{0}^{2 \pi} d \theta \operatorname{Tr} \mathcal{P}\left(U[A] \delta_{2} A_{a} \partial_{\theta} \bar{X}^{a}\right) \\
-\frac{1}{2} \alpha^{\prime 2} \int_{0}^{2 \pi} d \theta d \theta^{\prime} \operatorname{Tr} \mathcal{P}\left(U[A] \delta_{1} A_{a}(\bar{X}(\theta)) \partial_{\theta} \bar{X}^{a} \delta_{1} A_{b}\left(\bar{X}\left(\theta^{\prime}\right)\right) \partial_{\theta^{\prime}} \bar{X}^{b}\right)+\mathcal{O}\left(\alpha^{\prime 3}\right)
\end{gathered}
$$

Scale invariance of the theory is guaranteed by requiring the $\beta$-function for the Yang-Mills field to vanish:

$$
\beta_{a}=\beta_{a}^{i} t^{i}=\frac{\partial}{\partial(\ln \varepsilon)} \delta A_{a}=0
$$

this being interpreted as the equation of motion for $A_{a}$.

Contributions at the 1-loop level come from the $\mathcal{O}\left(\xi^{2}\right)$ terms in the expansion (10), giving the diagrams of Fig. 1. Fig. 1(b) is identically zero, since the derivative of the propagator with respect to the boundary variable $\theta$ is $\partial_{\theta} G(0)=0$. This leaves Fig. 1(a) alone, which generates

$$
\exp \left(-\triangle S_{1}\right)=i g \pi \alpha^{\prime} N_{d i v} \int_{0}^{2 \pi} d \theta \operatorname{Tr} \mathcal{P}\left(U[A] D^{b} F_{b a} \partial_{\theta} \bar{X}^{a}\right)
$$


$(a)$

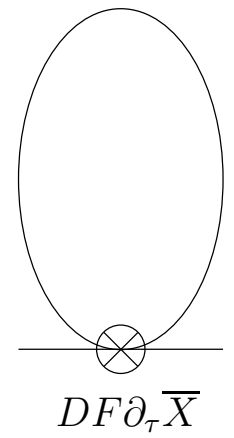

(b)

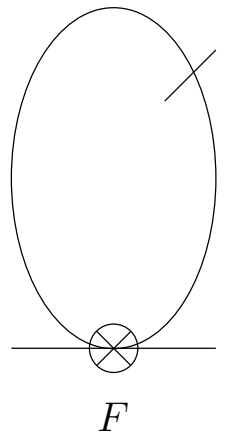

Figure 1. One-loop diagrams.

The solid loops represent a bosonic propagator; and a slash, the derivative of the propagator.

Due to the symmetry properties invloved, (b) is identically zero.

Comparison with (13) implies

$$
\delta A_{a}^{i}=\alpha^{\prime} g D^{b} F_{b a}^{i} \ln (\varepsilon)+\mathcal{O}\left(\alpha^{\prime 2}\right)
$$

and so

$$
\beta_{a}^{i}=\alpha^{\prime} g D^{b} F_{b a}^{i}+\mathcal{O}\left(\alpha^{\prime 2}\right) .
$$

To lowest order, then, $\beta_{a}^{i}=0$ is just the familiar Yang-Mills field equation.

At the two-loop level, there are two distinct contributions: those terms through $\mathcal{O}\left(\xi^{4}\right)$ in (10); but also those through $\mathcal{O}\left(\xi^{2}\right)$ in an iterative expansion of the first order counterterm (14). Again ignoring $\mathcal{O}(\xi)$ terms, the latter is given by

$$
\begin{aligned}
\exp \left(-\triangle S_{1}[\bar{X}+\xi]\right) & =i g \pi \alpha^{\prime} \frac{1}{2} N_{d i v} \int_{0}^{2 \pi} d \theta \operatorname{Tr} \mathcal{P}\left(U [ A ] \left[\left(D_{a} D^{c} F_{c b}-D_{b} D^{c} F_{c a}\right) \xi^{a} \partial_{\theta} \xi^{b}\right.\right. \\
& \left.\left.+\left(D_{b} D_{d} D^{c} F_{c a}-D_{a} D_{b} D^{c} F_{c d}\right) \xi^{b} \xi^{d} \partial_{\theta} \bar{X}^{a}\right]\right)
\end{aligned}
$$

which generates the on-shell diagrams of Fig. 2. Fig. 2(b) being identically zero, we are left with

$$
\begin{gathered}
\exp (- \text { Fig. } 2(\mathrm{a}))= \\
-i g\left(\pi \alpha^{\prime}\right)^{2} N_{d i v} \int_{0}^{2 \pi} d \theta N(0) \operatorname{Tr} \mathcal{P}\left(U[A]\left[D^{2} D^{b} F_{b a}-D^{c} D_{a} D^{b} F_{b c}+i g\left[D^{b} F_{b c}, F_{a}^{c}\right]\right] \partial_{\theta} \bar{X}^{a}\right),
\end{gathered}
$$

where the relation $\left[D_{a}, D_{b}\right] F_{c d}=-i g\left[F_{a b}, F_{c d}\right]$ has been used. 
(a)

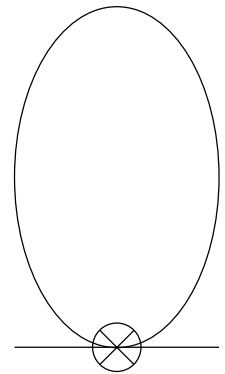

$(D D F-D D F) \partial_{\tau} \bar{X}$ $(b)$

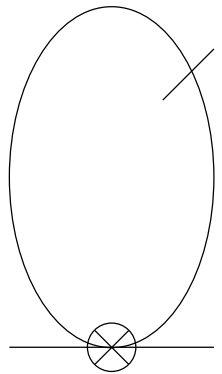

$(D D D F-D D D F+[D, D] D F)$

Figure 2. Two-loop diagrams.

Diagrams generated from an iterative expansion of the first order counterterm.

Due to the symmetry properties involved, (b) is identically zero.

The two-loop diagrams generated by (10) are shown in Fig. 3. Note that the $\mathcal{O}\left(\xi^{3}\right)$ terms do not contribute at all, since we have consistently dropped the $\mathcal{O}(\xi)$ terms. Then

$$
\begin{gathered}
\exp (- \text { Fig. } 3(\mathrm{a}))= \\
i g \frac{1}{6}\left(\pi \alpha^{\prime}\right)^{2} \int_{0}^{2 \pi} d \theta[N(0)]^{2} \operatorname{Tr} \mathcal{P}\left(U[A]\left[D^{2} D^{b} F_{b a}+D^{c} D^{b} D_{c} F_{b a}+D^{b} D^{2} F_{b a}\right] \partial_{\theta} \bar{X}^{a}\right) ;
\end{gathered}
$$

and

$$
\begin{gathered}
\exp (- \text { Fig. } 3(\mathrm{~b}))= \\
\left(\alpha^{\prime} g\right)^{2} \int_{0}^{2 \pi} d \theta\left(\frac{\pi^{2}}{4}[N(0)]^{2}+\ln (\varepsilon)\right) \operatorname{Tr} \mathcal{P}\left(U[A]\left[D_{a} F_{b c}, F^{b c}\right] \partial_{\theta} \bar{X}^{a}\right),
\end{gathered}
$$

all others being identically zero. Due to the symmetry properties of the region around $\theta \rightarrow \theta^{\prime}$, this latter would be zero in the abelian case but is non-zero for the case in hand.

Adding (18), (19) and (20) we get the total 2-loop counterterm

$$
\begin{gathered}
\exp \left(-\triangle S_{2}\right)= \\
-i g\left(\pi \alpha^{\prime}\right)^{2} N_{d i v}^{2} \int_{0}^{2 \pi} d \theta \operatorname{Tr} \mathcal{P}\left(U[A]\left[-\frac{1}{2} D^{2} D^{b} F_{b a}+\frac{4}{3} D^{c} D_{a} D^{b} F_{b c}-i g\left[D^{b} F_{b c}, F_{a}^{c}\right]\right] \partial_{\theta} \bar{X}^{a}\right) \\
-i g\left(\pi \alpha^{\prime}\right)^{2} N_{d i v} \int_{0}^{2 \pi} d \theta N_{f i n} \operatorname{Tr} \mathcal{P}\left(U[A]\left[\frac{5}{3} D^{c} D_{a} D^{b} F_{b c}-i g\left[D^{b} F_{b c}, F_{a}^{c}\right]\right] \partial_{\theta} \bar{X}^{a}\right) \\
-\left(\alpha^{\prime} g\right)^{2} \ln (\varepsilon) \int_{0}^{2 \pi} d \theta \operatorname{Tr} \mathcal{P}\left(U[A]\left[D_{a} F_{b c}, F^{b c}\right] \partial_{\theta} \bar{X}^{a}\right),
\end{gathered}
$$

where we have used the relation $D^{2} F_{a b}=D_{a} D^{c} F_{c b}-D_{b} D^{c} F_{c a}+2 i g\left[F_{a c}, F_{b}^{c}\right]$. There are two points about (21) which must be considered: firstly, the $N_{\text {div }}^{2}$ term does not contribute

\footnotetext{
${ }^{4}$ The evaluation of Fig. 3(b). is somewhat involved; we owe the calculation to that of Dorn and Otto in 110$]$.
} 
to the $\beta$-function at all, else it would itself be divergent; and, secondly, since (16) gives the equation of motion $D^{a} F_{a b}^{i}=\mathcal{O}\left(\alpha^{\prime}\right)$, the $N_{d i v} N_{\text {fin }}$ term is effectively of $\mathcal{O}\left(\alpha^{\prime 3}\right)$ and so can be dropped.

(a)

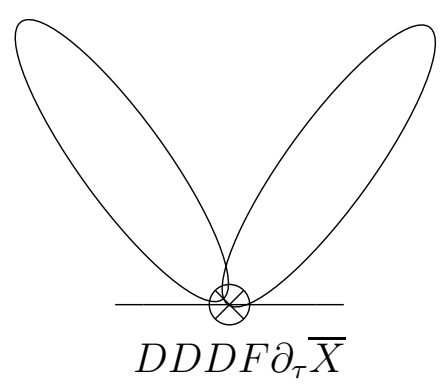

(c)

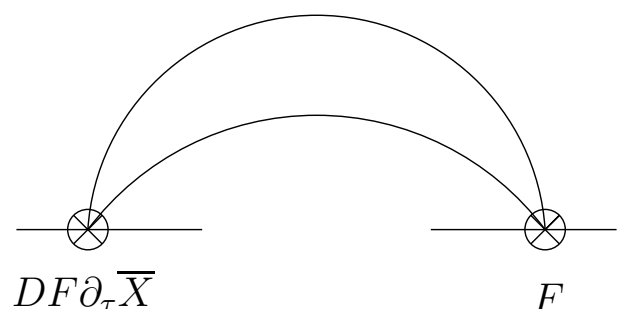

(b)

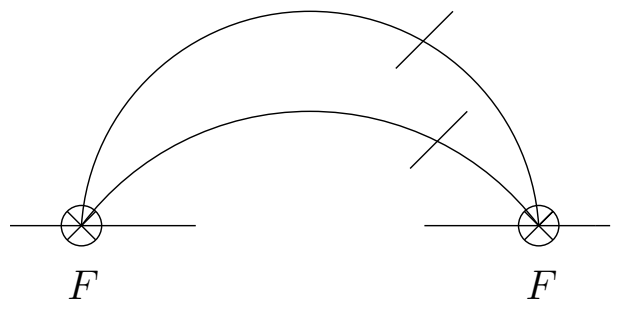

$(d)$

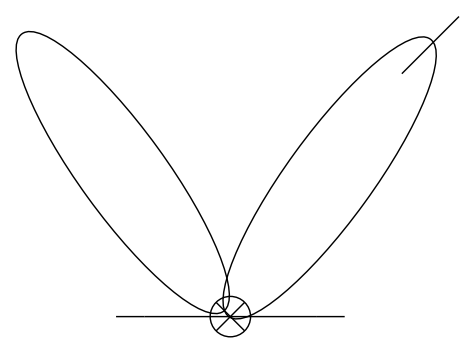

$D D F$

Figure 3. Two-loop diagrams.

Due to the symmetry properties involved, (c) and (d) are identically zero.

Ignoring the first two terms in (21), then, and comparing with (13), we obtain the same 2-loop counterterm as [10]:

$$
\delta A_{a}=\left(\alpha^{\prime} g D^{b} F_{b a}+i\left(\alpha^{\prime} g\right)^{2}\left[D_{a} F_{b c}, F^{b c}\right]\right) \ln (\varepsilon)+\mathcal{O}\left(\alpha^{\prime 3}\right) .
$$

For a semisimple, compact group with $\left[t^{i}, t^{j}\right]=i c^{i j k} t^{k}$, we have

$$
\begin{aligned}
\beta_{a}^{i} & =\alpha^{\prime} g D^{b} F_{b a}^{i}-\left(\alpha^{\prime} g\right)^{2} c^{i j k}\left(D_{a} F_{b c}^{j}\right) F^{k b c}+\mathcal{O}\left(\alpha^{\prime 3}\right) \\
& =\alpha^{\prime} g D^{b} F_{b a}^{i}-2\left(\alpha^{\prime} g\right)^{2} c^{i j k} D^{b}\left(F_{b c}^{j} F_{a}^{k c}\right)+\mathcal{O}\left(\alpha^{\prime 3}\right),
\end{aligned}
$$

where we have used the Bianchi identity and have again dropped terms like $D^{a} F_{a b}^{i} \sim \mathcal{O}\left(\alpha^{\prime}\right)$. By setting $\beta_{a}^{i}=0$, we obtain the equation of motion for the Yang-Mills background gauge field, viz.

$$
D^{b} F_{b a}^{i}-2 \alpha^{\prime} g c^{i j k} D^{b}\left(F_{b c}^{j} F_{a}^{k c}\right)=0,
$$

the lowest order stringy correction to the Yang-Mills field equation, at least within the bosonic theory. We will see that for the open superstring, the $\mathcal{O}\left(\alpha^{\prime 2}\right)$ term in the $\beta$-function vanishes, the fermionic contribution cancelling the two-loop bosonic divergence. 


\section{$3.2 \quad$ The Open Superstring}

We have found the first order correction to the Yang-Mills equation for the spacetime gauge field within open bosonic string theory. Since this is precisely how the results which led to the BI action were found, we should expect to be able to infer a non-abelian generalisation of the BI (and the DBI) action from these calculations. However, if we are to apply our results to D-branes we really should consider the $\beta$-functions for the open superstring, since the D-brane is embedded in the supersymmetric theory. We have mentioned above that, in the abelian case, the BI action is valid for the superstring, as well as in the bosonic case [16]. In the non-abelian case at hand, however, it is not at all obvious that the fermionic degrees of freedom do not contribute to the $\beta$-function.

Following [16], then, we define the fermionic propagator, $K^{a b}\left(z, z^{\prime}\right)$, in a manner analogous to the bosonic one: on the boundary, $K^{a b}\left(e^{i \theta}, e^{i \theta^{\prime}}\right)=-2 \pi \alpha^{\prime} \delta^{a b} K\left(e^{i \theta}, e^{i \theta^{\prime}}\right)$ where we take

$$
K\left(e^{i \theta}, e^{i \theta^{\prime}}\right)=-\frac{1}{\pi} \sum_{r=1 / 2}^{\infty} \sin r \beta e^{-\varepsilon r},
$$

the sum being over the half-integers since the fermionic variable $\psi^{a}$ should be antiperiodic on the boundary of the disc. Since we are dropping all $\mathcal{O}(\xi)$ terms in the expansion (10), there are only three extra fermionic diagrams to consider: those shown in Fig. 4. For the same reason that Fig. 3(b) is identically zero, so is the one-loop diagram Fig. 4(a): $K(0) \sim \sin (0)=0$. There is thus no further contribution at the one-loop level. It is for this simple reason that the results of [6, 7], for the abelian case, carry through to the supersymmetric theory. The analysis of these papers is at the one-loop level only, although is exact at this level for a slowly-varying field strength; within this approximation, then, the fermionic degrees of freedom simply do not contribute, there being no non-zero one-loop fermionic diagrams.

Since we are working to the two-loop order, our results will be valid not just to lowest order in derivatives of the field strength (although now, of course, these results will not be exact in $\alpha^{\prime}$ ). The two-loop fermionic diagrams of Fig. 4 must, then, be taken into account. Fig. 4(b) is once again zero, so we are left with Fig. 4(c) alone. Now, it is easy to see that for the region of interest, $\theta \rightarrow \theta^{\prime}$, we have that $K^{2} \sim\left(\partial_{\theta} N\right)^{2}$, as should be expected from supersymmetry considerations, and it is for this reason that

$$
\exp (- \text { Fig. } 4(\mathrm{c}))=-\left(\alpha^{\prime} g\right)^{2} \ln (\varepsilon) \int_{0}^{2 \pi} d \theta \operatorname{Tr} \mathcal{P}\left(U[A]\left[D_{a} F_{b c}, F^{b c}\right] \partial_{\theta} \bar{X}^{a}\right),
$$


which exactly cancels the logarithmic divergence in (20). After adding (26) to the two-loop counterterm (21) and dropping the $N_{d i v}^{2}$ and $N_{d i v} N_{\text {fin }}$ terms as before we find, then, that

$$
\begin{gathered}
\exp \left(-\triangle S_{2}\right)_{\text {total }}=0 \Rightarrow \\
\delta A_{a}^{i}=\alpha^{\prime} g D^{b} F_{b a}^{i} \ln (\varepsilon)+\mathcal{O}\left(\alpha^{\prime 3}\right) \Rightarrow \\
\beta_{a}^{i}=\alpha^{\prime} g D^{b} F_{b a}^{i}+\mathcal{O}\left(\alpha^{\prime 3}\right) .
\end{gathered}
$$

That is, the Yang-Mills equation is exact to $\mathcal{O}\left(\alpha^{\prime}\right)$, a fact that has far-reaching ramifications for the non-abelian generalisation of the BI action.

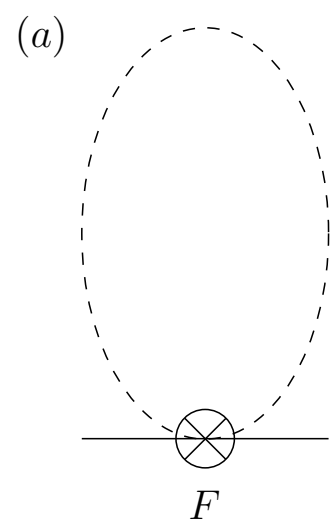

(b)

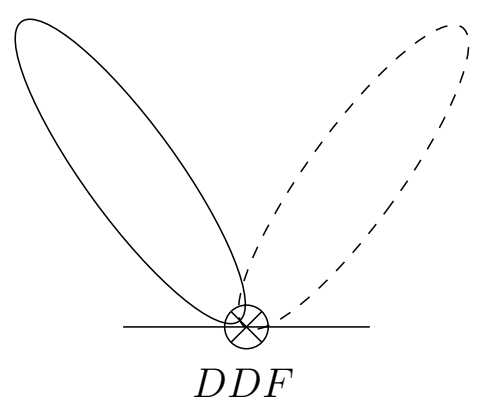

$(c)$

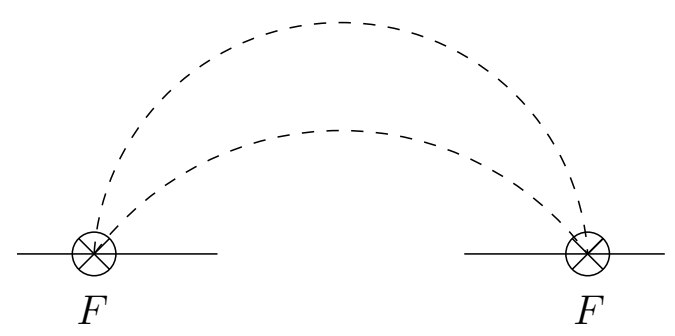

Figure 4. One- and two-loop diagrams fermionic contributions.

The dashed line represents the fermionic propagator.

Due to the symmetry properties involved, (a) and (b) are identically zero.

It should be noted that during the preparation of this paper, it has come to our attention that this result viz., the cancellation of the $\mathcal{O}\left(\alpha^{\prime 2}\right)$ term, has been verified previously using superspace techniques in [21, 22]. Indeed, three-loop diagrams are considered in this work, the implications of which will be discussed below. 


\section{The Non-Abelian Born-Infeld Action}

Our aim here is to find some non-abelian generalisation of the BI action (5), the equation of motion of which will have the same solutions as the equation $\beta_{b}^{i}=0$, with $\beta_{b}^{i}$ given in (27). We have shown this latter to be just the usual Yang-Mills field equation, so it would seem that we do not have much to go on. This is not true, however: the obvious first step is to simply replace the field strength in (5) with its non-abelian generalisation $F_{a b}^{i} t^{i}$, and to replace the metric with $\delta_{a b} \mathcal{I}, \mathcal{I}$ the unit matrix over group space; giving $\sqrt{\operatorname{det}\left(\delta_{a b} \mathcal{I}+2 \pi \alpha^{\prime} g F_{a b}^{i} t^{i}\right)}$. Then, since the lagrangian must be a scalar, both in spacetime as well as in the group, we must include some trace (or determinant) operation over the group indices. There has been some recent [1], and some not so recent [9], work which suggests two different such trace operations; we will show that our results for the superstring support the former.

We can immediately rule out certain possible generalisations of the BI action. That is, consider diagonalising the field strength, $F_{a b}^{i} t^{i}=\operatorname{diag}\left(F_{a b}^{1}, F_{a b}^{2}, \ldots, F_{a b}^{N}\right)$, and then performing the relevant trace operation. We should thereby obtain the sum of $N$ (ten-dimensional) BI actions. In the dimensionally reduced $\mathrm{T}$-dual theory, to be discussed below, this would correspond to $N$ separated and parallel D $p$-branes. Now it is easy to see that, only if the group trace operation occurs outside of the square root, will this be the case. On these very general grounds we must, then, have something of the form

$$
\mathcal{L}_{N B I}=\left(2 \pi \alpha^{\prime} g\right)^{-2} \operatorname{Tr}\left[\mathcal{I}-\sqrt{\operatorname{det}\left(\delta_{a b} \mathcal{I}+2 \pi \alpha^{\prime} g F_{a b}\right)}\right]
$$

We can thus immediately exclude lagrangians with different group trace structures, such as $\sqrt{\operatorname{Tr} \operatorname{det}\left(\delta_{a b} \mathcal{I}+2 \pi \alpha^{\prime} g F_{a b}\right)}$, which was indeed proposed by Hagiwara 23, although not in the context of D-brane theory, or $\sqrt{\operatorname{Det} \operatorname{det}\left(\delta_{a b} \mathcal{I}+2 \pi \alpha^{\prime} g F_{a b}\right)}$, Det a determinant over group space. Indeed, the analysis of [9] also excludes such lagrangians Moreover, this latter work also excludes the obvious trace operation in (28). In the light of these results, then, the only possible action, modulo certain terms to be discussed below, is that proposed by Tseytlin in [1]:

$$
\mathcal{L}_{N B I}=\left(2 \pi \alpha^{\prime} g\right)^{-2} \operatorname{STr}\left[\mathcal{I}-\sqrt{\operatorname{det}\left(\delta_{a b} \mathcal{I}+2 \pi \alpha^{\prime} g F_{a b}\right)}\right]
$$

where $\operatorname{STr}\left(M_{1}, M_{2}, \ldots, M_{n}\right)=\frac{1}{n !} \sum_{\pi} \operatorname{Tr}\left(M_{\pi(1)} M_{\pi(2)} \ldots M_{\pi(n)}\right)$ denotes a symmetrised group

\footnotetext{
${ }^{5}$ In [9], the low-energy effective action for an open bosonic string charged under a $U(1)$ subgroup of the full-blown $U(N)$ Yang-Mills background field is considered. The effective action is inferred via BRST invariance, and specifically excludes such terms as $\left(\operatorname{Tr} F^{2}\right)^{2}$, which would be generated if the group trace were to appear inside of the square root.
} 
trace. Note that we can further define an antisymmetrised group trace in a similar fashion: $\operatorname{ATr}\left(M_{1}, M_{2}, \ldots, M_{n}\right)=\frac{1}{n !} \sum_{\pi}(-1)^{\pi} \operatorname{Tr}\left(M_{\pi(1)} M_{\pi(2)} \ldots M_{\pi(n)}\right)$. For reasons to be discussed below, there is some ambiguity here as to which representation the group trace is to be taken over (cf. [1, 24]). Since the gauge field which couples to the ends of the open string in the boundary action (9) must be in the fundamental representation, however, the field strength in (29) must take values in this representation also. We will therefore require the symmetrised group trace to be taken over the fundamental representation.

A naïve variation of the above action, ignoring the matrix-ordering subleties, will just give the obvious generalisation of the equation of motion (4) for the abelian case (e.g. [22]):

$$
\frac{\delta \mathcal{L}_{N B I}}{\delta A_{a}^{i}}=\mathrm{S} \operatorname{Tr}\left[\sqrt{\operatorname{det}\left(\delta_{a b} \mathcal{I}+2 \pi \alpha^{\prime} g F_{a b}\right)}\left(\mathcal{I}-\left(2 \pi \alpha^{\prime} g\right) F^{2}\right)_{a}^{-1 b} \beta_{b} t^{i}\right]
$$

with $\beta_{a}=\alpha^{\prime}\left(\mathcal{I}-\left(2 \pi \alpha^{\prime} g F\right)^{2}\right)^{-1 b c} D_{(b} F_{c) a}$ the natural generalisation of the $\beta$-function for the abelian gauge field. Under the STr operation, it is clear that the different factors in (30) will become mixed; and it is not obvious that this equation of motion is equivalent to the statement $\beta_{a}^{i}=0$, as it is in the abelian case. To $\mathcal{O}\left(\alpha^{\prime 2}\right)$, however, and, indeed, to $\mathcal{O}\left(\alpha^{\prime 3}\right)$ 22] the two viewpoints are in fact equivalent.

At any rate, to this order, if we expand the spacetime determinant term-by-term it will be obvious that the equation of motion following from (29) is identical to the statement $\beta_{a}^{i}=0$. Using the identity $\operatorname{det} M=\exp (\operatorname{tr}(\ln M))$, with 'tr' a trace over spacetime indices, we generate a sum of powers of $F_{a b}$ :

$$
\begin{gathered}
\left(2 \pi \alpha^{\prime} g\right)^{-2}\left[\mathcal{I}-\sqrt{\operatorname{det}\left(\delta_{a b} \mathcal{I}+2 \pi \alpha^{\prime} g F_{a b}\right)}\right]= \\
-\frac{1}{2}\left[\frac{1}{2} F^{2}+\frac{1}{3}\left(2 \pi \alpha^{\prime} g\right) F^{3}+\frac{1}{16}\left(2 \pi \alpha^{\prime} g\right)^{2}\left(\left(F^{2}\right)^{2}-4 F^{4}\right)\right]+\mathcal{O}\left(\alpha^{\prime 3}\right),
\end{gathered}
$$

where $F^{2}=F_{a b} F^{a b}, F^{3}=F_{a b} F^{b c} F_{c}{ }^{a}=\frac{1}{2}\left[F_{a b}, F^{b c}\right] F_{c}{ }^{a}$ and $F^{4}=F_{a b} F^{b c} F_{c d} F^{d a}$. Now it is easy to see that, since $F_{a b}$ is antisymmetric in its spacetime indices, the symmetrised trace will pick out the even powers of $F_{a b}$ from this expansion only; and an antisymmetrised trace, the odd powers only. The lagrangian (29) thus contains even powers of $F_{a b}$ alone. Indeed, it is derived in [1] by assuming all odd powers of $F_{a b}$ to be negligible. Since $[D, D] F \sim$ $[F, F] F \sim F^{3}$, Tseytlin is led to assume the $F^{3}$ term (and all higher order odd invariants) in (31) to be negligible within, that is, the approximation that the field strength $F_{a b}$ is slowly varying. Moreover, this 'slowly varying limit' is just an 'abelian limit', in which the 
matrices $F_{a b}$ are assumed to commute. It is this 'abelian approximation' which is imposed by symmetrising the group trace in (28) to give (29).

The question is, then, whether odd powers (or derivatives) of $F_{a b}$ should contribute to the non-abelian BI action. The analysis of [9], in which it was found to be necessary to introduce $F^{3}$ terms into the effective action, suggests that they should; this being achieved by replacing $\mathrm{STr}$ with $(\mathrm{STr}+i \mathrm{ATr})$ in (29). These results are for the bosonic string, however. Indeed, our above analysis of the bosonic case supports precisely this conclusion: the $D^{b}\left(F_{b c} F_{a}^{c}\right)$ term in (24) is just what would follow from a variation of an $F^{3}$ term in the non-abelian BI action.

In the supersymmetric case, however, this term cancels with fermionic contributions so, since the $F^{3}$ term cannot appear in the non-abelian BI action, we must have STr as the correct trace operation. A three-loop analysis will contribute at $\mathcal{O}\left(\alpha^{\prime 3}\right)$, giving terms which should follow from the $F^{4}$ terms in (29):

$$
\begin{gathered}
\mathcal{L}_{N B I}=-\frac{1}{2}\left[\frac{1}{2} \operatorname{Tr}\left(F^{2}\right)+\right. \\
\left.+\frac{1}{48}\left(2 \pi \alpha^{\prime}\right)^{2} \operatorname{Tr}\left(F_{a b} F_{c d} F^{a b} F^{c d}+2 F_{a b} F^{a b} F_{c d} F^{c d}-4 F_{a b} F^{b c} F_{c d} F^{d a}-8 F_{a b} F^{b c} F_{a d} F_{c}^{d}\right)\right] .
\end{gathered}
$$

Up to an overall factor, the coefficients of the $F^{2}$ and $F^{4}$ terms match those found via stringy calculations in [16], in which the absence of $F^{3}$ terms for the effective action of the superstring is further confirmed. To $\mathcal{O}\left(\alpha^{\prime}\right)$, (32) just implies the Yang-Mills equation, identical to $\beta_{b}^{i}=0$ in (27). This we take as our justification for the non-abelian Born-Infeld action (29). The bottom line is that both $\operatorname{Tr}$ and ATr, when applied to the obvious generalisation of the BI action will give $F^{3}$ terms (and higher order odd invariants). The fact that, at least the $F^{3}$ term should not appear, implies that we must take STr.

As mentioned above, a three-loop analysis is undertaken in [21, 22], in which it is verified that the $F^{3}$ term should be absent. Moreover, it is shown that the $\mathcal{O}\left(\alpha^{\prime 3}\right)$ terms in the $\beta$ function agree with the equation of motion following from (32). To this order, then, the action (29) is correct, as indeed is the view taken that the vanishing of the $\beta$-function is equivalent to stationarity of this action.

Our analysis suggests also an extension to the region of validity of (29), over and above that assumed in [1], in that we have shown the terms of lowest order in derivatives of the field strength to be absent from the non-abelian BI action; this latter should be correct not just for a slowly-varying field strength. Whether all higher order odd invariants should vanish, the action depending solely on non-derivative terms, is a question we cannot answer in the 
approach taken here without, that is, performing higher-loop calculations.

\subsection{T-duality, D-branes and the Mass Term}

To see the appearance of $\mathrm{D}$-branes we must consider applying $\mathrm{T}$-duality to our effective spacetime theory (29). That is, we again consider compactifying the theory on a circle of radius $R$ in the $X^{9}$ direction [4]. Then, since the field $A_{9}$ must be pure gauge, we have the Wilson line $A_{9}=-i \Lambda^{-1} \partial_{9} \Lambda=\operatorname{diag}\left\{\theta_{1}, \theta_{2}, \ldots, \theta_{N}\right\} /(2 \pi R)$ and, under $X^{9} \rightarrow X^{9}+2 \pi R$,

the embeddings pick up a phase $\operatorname{diag}\left\{e^{-i \theta_{1}}, e^{-i \theta_{2}}, \ldots, e^{-i \theta_{N}}\right\}$. In the dual theory, we have $\tilde{X}^{9}(\pi)-\tilde{X}^{9}(0)=\left(2 \pi n+\theta_{j}-\theta_{i}\right) / \tilde{R}$, for a string with endpoints in the state $|i j\rangle$; up to a normalisation, the endpoint in the state $i$ is at the position $\tilde{X}^{9}=\theta_{i} \tilde{R}=2 \pi \alpha^{\prime} A_{9, i i}$. This is just a theory of $N$ separated, and parallel, D8-branes on the dual circle, the diagonal components of $A_{9}$ specifying the positions of the branes along this circle.

Following [11], it is to be understood that the dual coordinate $\tilde{X}^{9}$, interpreted as a fullblown group matrix, specifies the transverse position of the generic bound state of the $N$ D8-branes, at least when they are all sitting on top of each other. The process by which the spacetime coordinates of such a 'brane' become matrix-valued is by no means clear, however. Perhaps, if we were to compactify the ten-dimensional $U(N)$ theory on a manifold with a natural non-abelian structure (rather than the trivial circle as above), some light might be shed on this. Just as compactification on a circle gives a theory of $N$ separated and parallel D8-branes, so compactification on a manifold with, say, an $S U(2)$ structure should give a theory of $N / 2$ separated and parallel bound states of two D8-branes. These latter will have matrix-valued coordinates, the emergence of which might become clearer by this process.

At any rate, we will simply suppose some compact directions, in which the coordinates are assumed to be matrix-valued: $X^{\alpha}=2 \pi \alpha^{\prime} A^{\alpha}, \quad \alpha=p+1, \ldots, 9$, where we have dropped the tilde from the dual coordinate. For $A, B=0,1, \ldots, p$ denoting worldvolume directions, we have $F_{A \alpha}=\left(2 \pi \alpha^{\prime}\right)^{-1}\left(\partial_{A} X_{\alpha}-i\left[A_{A}, X_{\alpha}\right]\right)=\left(2 \pi \alpha^{\prime}\right)^{-1} D_{A} X_{\alpha}$ and $F_{\alpha \beta}=-i\left(2 \pi \alpha^{\prime}\right)^{-2}\left[X_{\alpha}, X_{\beta}\right]$, since all fields are taken to depend on the worldvolume coordinates alone (and where the gauge coupling constant $g$ has now been set to unity). Then using the identity (6), e.g. [1]:

$$
\begin{gathered}
\mathcal{L}_{N B I}=\left(2 \pi \alpha^{\prime}\right)^{-2} \operatorname{STr}\left[\mathcal{I}-\sqrt{\operatorname{det}\left(\delta_{\alpha \beta} \mathcal{I}-i\left(2 \pi \alpha^{\prime}\right)^{-1}\left[X_{\alpha}, X_{\beta}\right]\right)}\right. \\
\left.\times \sqrt{\operatorname{det}\left(\delta_{A B} \mathcal{I}+D_{A} X_{\alpha}\left(\delta_{\alpha \beta}-i\left(2 \pi \alpha^{\prime}\right)^{-1}\left[X_{\alpha}, X_{\beta}\right]\right)^{-1} D_{B} X_{\beta}+2 \pi \alpha^{\prime} F_{A B}\right)}\right] .
\end{gathered}
$$


This is the lagrangian relevant to the description of bound states of $\mathrm{D} p$-branes. To lowest order in $\left[X_{\alpha}, X_{\beta}\right]$, we have

$$
\begin{aligned}
\mathcal{L}_{N B I}=\left(2 \pi \alpha^{\prime}\right)^{-2} \operatorname{STr} & {\left[\mathcal{I}-\left(\sqrt{\operatorname{det}\left(\delta_{A B} \mathcal{I}+D_{A} X_{\alpha} D_{B} X^{\alpha}+2 \pi \alpha^{\prime} F_{A B}\right)}\right.\right.} \\
& \left.\left.-\frac{1}{4}\left(2 \pi \alpha^{\prime}\right)^{-2}\left(\left[X_{\alpha}, X_{\beta}\right]\right)^{2}\right)\right]
\end{aligned}
$$

As is to be expected, in the low-amplitude limit, the action (34) is just the reduction from ten to $(p+1)$ dimensions of the ten-dimensional $U(N)$ Yang-Mills theory, the potential $V=\frac{1}{4}\left(2 \pi \alpha^{\prime}\right)^{2}\left(\left[X_{\alpha}, X_{\beta}\right]\right)^{2}$.

Since $N$ parallel $\mathrm{D} p$-branes will interact via massive string states, it is an interesting question as to whether this mass term will appear within the non-abelian theory (34). To this end we assume the potential $V$ to vanish, in which case we can simultaneously diagonalise the transverse coordinates $X^{\alpha}$, the diagonal entries specifying the position of each brane: $X^{\alpha}=\operatorname{diag}\left(X_{1}^{\alpha}, X_{2}^{\alpha}, \ldots, X_{N}^{\alpha}\right)$. And this is where the ambiguity arises as to which representation the symmetrised trace is to be taken over. It is usual to take the scalars in the dimensionally reduced Yang-Mills theory to lie in the adjoint representation, but we have stated above that the field strength, from which they appear, lies in the fundamental. Now consider the $S U(2)$ theory, which by rights should describe a bound state of two $\mathrm{D} p$-branes, the entries in the diagonalised $X_{\alpha}$ 's corresponding to the positions of the two parallel and separated branes. If we were to take $X_{\alpha}$ to lie in the adjoint, it would be a $3 \times 3$ matrix, which surely cannot correspond in any way to just two branes. If we consider the fundamental representation, however, then our interpretation is consistent: the diagonal elements of the $2 \times 2$ matrices $X_{\alpha}$ can correspond to the positions of two parallel branes. Our statement above, then, that the symmetrised trace must be taken over the fundamental representation, would seem to be consistent.

With the transverse coordinates diagonalised, we have $V=0$; and there are two distinct cases to consider. Firstly, we can further diagonalise the worldvolume field strength, as above. Then $D_{A} X^{\alpha} \rightarrow \partial_{A} X^{\alpha}$ and $\mathrm{STr} \rightarrow \mathrm{Tr}$, since everything is diagonal. The action (34) then reduces to the direct sum of $N$ DBI actions, with the usual interpretation of $N$ separate and parallel Dp-branes. There is no mass term here.

A more interesting case would be to keep the field strength truly non-abelian. Then, although we should still have the $N$ separate and parallel $\mathrm{D} p$-branes (the transverse embeddings are still diagonal), we should also see the interactions between the branes emerging. 
We assume the branes to be stationary, in time and space, so $D_{A} X_{\alpha}=-i\left[A_{A}, X_{\alpha}\right]$ where the world-volume gauge potential now has off-diagonal components. The simplest case is that of two $\mathrm{D} p$-branes with one transverse coordinate excited: $X=\operatorname{diag}\left(X_{1}, X_{2}\right)$. The relevant gauge group, ignoring the overall abelian factor, is $S U(2)$. Then

$$
D_{A} X=-i(\triangle L) A_{A}^{i}\left(\begin{array}{cc}
0 & t_{12}^{i} \\
-t_{21}^{i} & 0
\end{array}\right)=-i \frac{(\triangle L)}{\sqrt{2}}\left(\begin{array}{cc}
0 & W_{A}^{*} \\
-W_{A} & 0
\end{array}\right),
$$

with $t^{i}=\sigma^{i} / 2, \operatorname{Tr}\left(t^{i} t^{j}\right)=\delta^{i j} / 2 . \triangle L=X_{2}-X_{1}$ is the separation of the branes (the length of the strings between them), and we have set $W_{A}^{*}=\left(A_{A}^{1}-i A_{A}^{2}\right) / \sqrt{2}$ and $W_{A}=\left(A_{A}^{1}+i A_{A}^{2}\right) / \sqrt{2}$. Expanding the determinant in (34) to lowest order gives

$$
\begin{aligned}
\mathcal{L}_{N B I}= & \left(2 \pi \alpha^{\prime}\right)^{-2} \operatorname{STr}\left[\mathcal{I}_{2}-\left(\mathcal{I}_{2}+D_{A} X D^{A} X+\frac{1}{2}\left(2 \pi \alpha^{\prime}\right)^{2} F^{2}\right.\right. \\
& \left.\left.-\frac{1}{2}\left[D_{A} X D_{B} X F^{B A}+F^{A B} D_{B} X D_{A} X\right]\right)^{1 / 2}\right] .
\end{aligned}
$$

Substituting for $D_{A} X D^{A} X=\left((\triangle L)^{2}|W|^{2}\right) / 2 \mathcal{I}_{2}$, where $|W|^{2}=W^{A} W_{A}^{*}=\left(A_{A}^{1} A^{1 A}+\right.$ $\left.A_{A}^{2} A^{2 A}\right) / 2$, and to lowest order, we have

$$
\begin{aligned}
\mathcal{L}_{N B I} & =-\frac{1}{4} \operatorname{Tr}\left(F^{2}\right)-\frac{1}{2} \frac{(\triangle L)^{2}}{\left(2 \pi \alpha^{\prime}\right)^{2}}|W|^{2} \\
& =-\frac{1}{4} \operatorname{Tr}\left(F^{2}\right)-\frac{1}{2} M^{2}|W|^{2}
\end{aligned}
$$

since $\operatorname{STr}\left(t^{i} t^{j}\right)=\operatorname{Tr}\left(t^{i} t^{j}\right)$ and $\operatorname{STr}\left(\mathcal{I}_{2}\right)=\operatorname{Tr}\left(\mathcal{I}_{2}\right)=2 . \quad M=(\triangle L) /\left(2 \pi \alpha^{\prime}\right)$ is the mass of the string stretching between the two branes. This analysis follows through with more than one compact direction, in which case $(\triangle L)^{2}=\left(X_{\alpha}^{2}-X_{\alpha}^{1}\right)\left(X^{2 \alpha}-X^{1 \alpha}\right)$. Note that the cross term $\left[D_{A} X D_{B} X F^{B A}+F_{A B} D^{B} X D^{A} X\right]$ drops out under the $\mathrm{STr}$ operation. The equations of motion are thus

$$
\left.\begin{array}{l}
D^{A} F_{A B}^{3}=0 \\
D^{A} F_{A B}^{i}=M^{2} A_{B}^{i}
\end{array}\right\}
$$

where now $i=1,2$ only. We can see that the mass term has appeared in the form of a massive Yang-Mills theory in the $\{1,2\}$ group directions. As should be expected, in this case gauge invariance is not realised in any obvious way.

There is a nice check of this result: following [25], we can consider applying T-duality to the $\beta$-function itself. With $\beta_{b}=\alpha^{\prime} D^{a} F_{a b}$ and $X^{9} \equiv X=2 \pi \alpha^{\prime} A^{9}$, we have

$$
\beta_{B}=\alpha^{\prime} D^{A} F_{A B}+\frac{i}{4 \pi^{2} \alpha^{\prime}}\left[X, D_{B} X\right]=0
$$




$$
\beta_{9}=\frac{1}{2 \pi} D^{A} D_{A} X=0,
$$

where, as above, we have assumed $X$ to be diagonalised. For static branes, we have

$$
\frac{1}{\sqrt{2}}\left(D^{A} F_{A B}\right)_{i j}-M^{2}\left(\begin{array}{cc}
0 & W_{B}^{*} \\
W_{B} & 0
\end{array}\right)=0 .
$$

This implies $D^{A} F_{A B}^{3}=0$ and $D^{A} F_{A B}^{i}=M^{2} A_{B}^{i}$ which are precisely the equations (37). To summarise, the application of $\mathrm{T}$-duality to the ten-dimensional $\beta$-function gives an equation of motion which, at least to lowest order, is just that obtained from the T-dual of the tendimensional non-abelian BI theory.

Moreover, we have shown how the non-abelian BI theory describes two parallel and interacting D-branes: to lowest order, this description is just that of a massive Yang-Mills field (in two of the group directions).

\subsection{The (Anti)-Self-Dual Case}

Unfortunately, an analysis of the non-abelian BI theory (29), or its dimensionally reduced version (34) in the generic case, and for anything but the lowest order terms, is a difficult problem. For multiple D4-branes with an (anti-)self-dual field strength, however, a considerable simplification occurs, due to the fact that the determinant can be written as a complete square. We consider the purely magnetic case, $F_{0 A}=0, A=1, \ldots, 4$. Then [26]

$$
\begin{aligned}
\operatorname{det}\left(\delta_{A B}+2 \pi \alpha^{\prime} F_{A B}\right) & =1+\frac{1}{4}\left(2 \pi \alpha^{\prime}\right)^{2} F^{2}+\frac{1}{2}\left(2 \pi \alpha^{\prime}\right)^{2} \tilde{F}^{2}+\frac{1}{16}\left(2 \pi \alpha^{\prime}\right)^{4}(F \cdot \tilde{F})^{2} \\
& =\left(1 \mp \frac{1}{4}\left(2 \pi \alpha^{\prime}\right)^{2} \operatorname{tr} F \tilde{F}\right)^{2}-\frac{1}{4}\left(2 \pi \alpha^{\prime}\right)^{2} \operatorname{tr}(F \mp \tilde{F})^{2}
\end{aligned}
$$

where $\tilde{F}_{A B}$ is the dual of $F_{A B}$. Setting all transverse coordinates to zero, and for an (anti)self-dual field strength, $F_{A B}= \pm \tilde{F}_{A B}$, we then have

$$
\mathcal{L}_{N B I}=\left(2 \pi \alpha^{\prime}\right)^{-2} \operatorname{STr}\left[\mathcal{I}-\sqrt{\left(\mathcal{I}+\frac{1}{4}\left(2 \pi \alpha^{\prime}\right)^{2} F^{2}\right)^{2}}\right]=-\frac{1}{4} \operatorname{Tr}\left(F^{2}\right),
$$

which is just the lagrangian for the linearised (Yang-Mills) theory. This has in fact already been noted in [27]. The self-duality condition is, as usual, solved by instanton configurations. In this case, then, the non-abelian BI action reduces to that of Yang-Mills theory. As explained in [26], the energy of solutions in this case is an absolute minima: they are BPS states. In fact, this will be the case for any situation in which the determinant can be written as a complete square. Most of the analysis of [26] will, then, carry through to the non-abelian 
case, at least for the BPS states themselves, for which the energy bound is saturated. It would seem, then, that the BPS states of the non-abelian BI theory are just those of its linearised version; it would be interesting to analyse this statement in more detail.

\section{Discussion}

The $\beta$-function for the non-abelian gauge field in open bosonic string theory suggests an extension of Tseytlin's recent proposal for the non-abelian BI action to be necessary. This is not true for the superstring, however; and since it is this latter case which is relevant to the study of bound states of D-branes, we effectively verify Tseytlin's proposal. We have shown how this proposal, and no other (no obvious others at least), gives an equation of motion compatible with the equation $\beta_{b}^{i}=0$ for the superstring. We have discussed the application of $\mathrm{T}$-duality to this action, giving the action relevant to bound states of D-branes; and have shown how the interaction between two separated and parallel branes appears in the theory. Although we have an action, it is hard to know quite what to do with it, in all but the simplest of cases. That is, when the determinant can be written as a complete square. For D4-branes, with an (anti-)self-dual field strength, the action reduces to the usual Yang-Mills action, the instanton solutions of which are then BPS states in the non-abelian BI theory. A detailed analysis of the action for D0-branes would be most welcome; this providing information as to corrections to the usual (dimensionally reduced) Yang-Mills action of M(atrix) theory.

\section{Acknowledgments}

One of the authors (DB) would like to thank Philip Argyres and Harald Dorn for electronic correspondence, Gary Gibbons and George Papadopoulos for enlightening discussions, and Miguel Costa and Steve Hewson for keeping me going. 


\section{A Appendix}

The expansion, (10), was derived in the early days of string theory when the role of the Wilson loop, providing a bridge between Yang-Mills and string theory, was emphasised; for a review see, e.g., [19]. We present here a derivation of the bosonic sector of (10) for completeness, following the analysis of [18]; the fermionic pieces follow easily. Consider, then, an arbitrary closed curve $X^{a}(\tau), 0 \leq \tau \leq 1$, and let $W[X]=\mathcal{P}\left(\exp i g \int_{0}^{1} A_{b}(X) d X^{b}\right)=\mathcal{P}(U[A])$. Then

$$
\begin{gathered}
W[\bar{X}+\xi]-W[\bar{X}]=\left.\int_{0}^{1} d \tau \frac{\delta W[X]}{\delta X^{a}(\tau)}\right|_{X=\bar{X}} \xi^{a}(\tau) \\
+\left.\frac{1}{2 !} \int_{0}^{1} d \tau_{1} \int_{0}^{1} d \tau_{2} \frac{\delta^{2} W[X]}{\delta X^{a}\left(\tau_{1}\right) \delta X^{b}\left(\tau_{2}\right)}\right|_{X=\bar{X}} \xi^{a}\left(\tau_{1}\right) \xi^{b}\left(\tau_{2}\right)+\mathcal{O}\left(\xi^{3}\right)
\end{gathered}
$$

To compute the functional derivatives, we expand the embedding

$$
X^{a}(\tau)=\lim _{M \rightarrow \infty} X_{(M)}^{a}(\tau)=\lim _{M \rightarrow \infty} \sum_{q=1}^{M} \alpha_{q}^{a} f_{q}(\tau),
$$

where

$$
\alpha_{q}^{a}=\int_{0}^{1} d \tau f_{q}(\tau) X^{a}(\tau)
$$

$\left\{f_{q}(\tau)\right\}$ are a set of 'equally dense' functions such that

$$
\begin{aligned}
f_{q}(\tau+1)=f_{q}(\tau) & , \quad \int_{0}^{1} d \tau f_{p}(\tau) f_{q}(\tau)=\delta_{p q}, \\
\sum_{q=1}^{M} f_{q}\left(\tau_{1}\right) f_{q}\left(\tau_{2}\right) & =\delta_{(M)}\left(\tau_{1}-\tau_{2}\right), \\
\lim _{M \rightarrow \infty} \frac{1}{M} \delta_{(M)}\left(\tau_{1}-\tau_{2}\right) & =\delta\left(\tau_{1}-\tau_{2}\right),
\end{aligned}
$$

Working in $\mathrm{D}$-dimensional spacetime, the functional $W[X]$ is considered as the limit of a function of $M \times D$ variables:

$$
W[X]=\lim _{M \rightarrow \infty} W_{(M)}\left(\alpha_{1}^{a_{1}} \ldots \alpha_{M}^{a_{M}}\right) \equiv \lim _{M \rightarrow \infty} W\left[X_{(M)}\right]
$$

and the functional derivatives are defined in terms of partial derivatives via

$$
\frac{\delta}{\delta X^{a}(\tau)}=\lim _{M \rightarrow \infty} \sum_{q=1}^{M} f_{q}(\tau) \frac{\partial}{\partial \alpha_{q}^{a}} .
$$

To compute the partial derivatives of $W[X]$, we must first expand the path ordered exponential:

$$
W[X]=i g \int_{0}^{1} d \tau A_{b}(X) \partial_{\tau} X^{b}+\frac{(i g)^{2}}{2} \int_{0}^{1} d \tau_{1} \int_{0}^{\tau_{1}} d \tau_{2}\left[A_{b}\left(X\left(\tau_{1}\right)\right), A_{c}\left(X\left(\tau_{2}\right)\right)\right] \partial_{\tau_{1}} X^{b} \partial_{\tau_{2}} X^{c}
$$




$$
+\frac{(i g)^{3}}{2} \int_{0}^{1} d \tau_{1} \int_{0}^{\tau_{1}} d \tau_{2} \int_{0}^{\tau_{2}} d \tau_{3} A_{b}\left(X\left(\tau_{1}\right)\right)\left[A_{c}\left(X\left(\tau_{2}\right)\right), A_{d}\left(X\left(\tau_{3}\right)\right)\right] \partial_{\tau_{1}} X^{b} \partial_{\tau_{2}} X^{c} \partial_{\tau_{3}} X^{d}+\mathcal{O}\left(e^{4}\right)
$$

needing the terms through $\mathcal{O}\left(g^{2}\right)$ to compute the first derivatives of $W[X]$, and the terms through $\mathcal{O}\left(g^{3}\right)$ for the second derivatives. We need compute the first partial derivative only, however, this being

$$
\frac{\partial W\left[X_{(M)}\right]}{\partial \alpha_{q}^{a}}=\mathcal{P}\left(U[A] i g \int_{0}^{1} d \tau f_{q} F_{a b}\left(X_{(M)}\right) \partial_{\tau} X_{(M)}^{b}\right)
$$

Using the definition (47), the property (45), and taking the limit $M \rightarrow \infty$ we then find

$$
\frac{\delta W[X]}{\delta X^{a}(\tau)}=\mathcal{P}\left(U[A] i g F_{a b}(X(\tau)) \partial_{\tau} X^{b}\right)
$$

The second functional derivative can be found easily from (50), by the repeated use of (47):

$$
\begin{gathered}
\frac{\delta^{2} W[X]}{\delta X^{b}\left(\tau_{2}\right) \delta X^{a}\left(\tau_{1}\right)}=\lim _{M \rightarrow \infty} \sum_{q=1}^{M} f_{q}\left(\tau_{2}\right) \frac{\partial}{\partial \alpha_{q}^{b}}\left(\frac{\delta W[X]}{\delta X^{a}\left(\tau_{1}\right)}\right)= \\
i g \mathcal{P}\left(U [ A ] \left[D^{b} F_{a c}\left(X\left(\tau_{1}\right)\right) \partial_{\tau_{1}} X^{c} \delta\left(\tau_{1}-\tau_{2}\right)+F_{a b} \partial_{\tau_{1}} \delta\left(\tau_{1}-\tau_{2}\right)\right.\right. \\
\left.\left.i e F_{a c}\left(X\left(\tau_{1}\right)\right) \partial_{\tau_{1}} X^{c} F_{b d}\left(X\left(\tau_{2}\right)\right) \partial_{\tau_{2}} X^{d}\right]\right),
\end{gathered}
$$

where the endpoint contributions (at $\tau=0,1$ ), which vanish anyway for a closed path, have been ignored. We note that the full non-abelian field strength and its covariant derivative appear in (50) and (51), respectively, due to the path ordering operation which introduces the relevant commutator terms, these being specific to the non-abelian case. Working to $\mathcal{O}(g)$, we now evaluate (50) and (51) at $X=\bar{X}$ and substitute into (42), giving

$$
\begin{gathered}
W[\bar{X}+\xi]-W[\bar{X}]= \\
i g \int_{0}^{1} d \tau \mathcal{P}\left(U[A]\left[F_{a b}(\bar{X}) \xi^{a} \partial_{\tau} \bar{X}^{b}+\frac{1}{2 !} D_{b} F_{a c}(\bar{X}) \xi^{a} \xi^{b} \partial_{\tau} \bar{X}^{c}+\frac{1}{2 !} F_{a b}(\bar{X}) \xi^{a} \partial_{\tau} \xi^{b}\right]\right) .
\end{gathered}
$$

To $\mathcal{O}\left(\xi^{2}\right)$, then, this is just the expansion (10), as promised. The higher order terms are generated by a straightforward generalisation of this result noting that, at each order, we will have two terms: one of the form $D \ldots D F \xi \ldots \xi \partial_{\tau} X$; and one of the form $F \xi \ldots \xi \partial_{\tau} \xi$. 


\section{References}

[1] A. A. Tseytlin. Nucl. Phys., B501:41, 1997. hep-th/9701125.

[2] J. Dai, R. G. Leigh, and J. Polchinski. Mod. Phys. Lett., A4:2073, 1989.

[3] J. Polchinski. Phys. Rev. Lett., 75:4724, 1995. hep-th/9510017.

[4] J. Polchinski. TASI Lectures on D-Branes. hep-th/9611050.

[5] M. Born and L. Infeld. Proc. Roy. Soc., A144:425, 1934.

[6] A. Abouelsaood, C. G. Callan, C. R. Nappi, and S. A. Yost. Nucl. Phys., B280:599, 1987.

[7] R. G. Leigh. Mod. Phys. Lett., A4:2767, 1989.

[8] H. Dorn. Nucl. Phys., B494:105, 1997. hep-th/9612120.

[9] P. C. Argyres and C. R. Nappi. Nucl. Phys., B330:151, 1990.

[10] H. Dorn and H.-J. Otto. Z. Phys., C32:599, 1986.

[11] E. Witten. Nucl. Phys., B460:335, 1996. hep-th/9510135.

[12] T. Banks, W.Fischler, S. H. Shenker, and L. Susskind. Phys. Rev., D55:5112, 1997. hep-th/9610043.

[13] C. G. Callan, D. Friedan, E. J. Martinec, and M. J. Perry. Nucl. Phys., B262:593, 1985.

[14] C. G. Callan and L. Thorlacius. Sigma Models and String Theory. In A. Jevicki and C.I. Tan, editors, Particles, Strings and Supernovae: Proc. TASI 1988, page 795. World Scientific, 1989.

[15] L. Alvarez-Gaumé and D. Z. Freedman. Ann. Phys., 134:85, 1981.

[16] A. A. Tseytlin. Nucl. Phys., B276, B291(E):391, 876(E), 1986, 1987(E).

[17] G. W. Gibbons. Nucl. Phys., B514:603, 1998. hep-th/9709027.

[18] J. L. Gervais and A. Neveu. Nucl. Phys., B153:445, 1979. 
[19] H. Dorn. Fortschr. Phys., 34:11, 1986.

[20] E. S. Fradkin and A. A. Tseytlin. Phys. Lett., 163B:123, 1985.

[21] K. Behrndt. Open Superstring in Non-Abelian Gauge Field. In H. J. Kaiser, editor, Proc. XXIII Int. Symp. Ahrenshoop 1989, page 174. Akademie der Wissenschaften der DDR, 1989.

[22] K. Behrndt. Untersuchung der Weyl-Invarianz im Verallgemeinerten $\sigma$-Modell für Offene Strings. PhD thesis, Humboldt-Universität zu Berlin, 1990.

[23] T. Hagiwara. J. Phys., A14:3059, 1981.

[24] G. W. Gibbons. Wormholes on the World Volume. hep-th/9801106.

[25] H. Dorn. Non-Abelian Gauge Field Dynamics on Matrix D-branes in Curved Space and Two-Dimensional $\sigma$-Models. hep-th/9712057.

[26] J. P. Gauntlett, J. Gomis, and P. K. Townsend. J. High Energy Phys., 01:003, 1998. hep-th/9711205.

[27] A. Hashimoto. Phys. Rev., D57:6441, 1998. hep-th/9711097. 